\title{
La qualité médicale est le fruit d'un dialogue
}

La qualité des soins médicaux est régulièrement au centre des discussions. Dans la politique de la santé, chez nos partenaires et au sein de la FMH. En tant que médecins, nous y portons une attention particulière car la qualité est intrinsèque à notre activité quotidienne et à notre éthique professionnelle; elle est même l'un des piliers porteurs de notre métier. A l'extérieur du corps médical, on dit fréquemment que rien n'est fait à ce sujet et qu'il faut définir des standards contraignants. De temps à autre, on pense même implicitement qu'en définissant des normes quelconques et en instituant des contrôles pointilleux, on œuvre en faveur de la qualité médicale.

La FMH considère que la qualité est une notion bien plus étendue que cela [1]. Il est indubitable que nos nombreux partenaires, avant tout les patients, ont droit à des soins médicaux de qualité. Il apparaît toutefois aussi que les parties en présence ont des idées sur la qualité qui divergent clairement. Par conséquent, la FMH estime nécessaire de conserver un aspect essentiel du débat sur ce sujet: celui de négocier avec tous les groupes d'interlocuteurs concernés. Le désir de certains partenaires de fixer unilatéralement des directives n'a pas de sens et est contreproductif. De surcroît, il convient de veiller à ne pas limiter les négociations à un seul cycle mais d'en faire un processus continuel, car la qualité est un élément dynamique par excellence. Malgré les ressources que cela requiert, la FMH est convaincue que seul un tel processus peut garantir une démarche de qualité judicieuse: elle a donc signalé à plusieurs reprises sa disponibilité à dialoguer.

En outre, c'est à bon droit que le corps médical rappelle régulièrement les nombreuses activités qu'il déploie depuis des années pour la promotion de la qualité. Nous agissons en sa faveur dans les cercles de qualité, dans l'annonce des fautes médicales, en instaurant et en développant des systèmes d'évaluation des cabinets médicaux ainsi que par la mise à disposition d'une excellente formation postgraduée et continue. Il s'agit, en l'occurrence, d'une combinaison d'initiatives multiples partant du niveau local pour aboutir à des projets internationaux, toutes servant à promouvoir la qualité d'une médecine axée sur les besoins des patients. Le défi à relever est sans conteste celui de rendre nos nombreuses activités compréhensibles et visibles pour le grand public et avant tout pour les patients.

Notre intention, ici, n'est pas de publier une déclaration unilatérale, mais d'indiquer simplement que le corps médical a beaucoup à offrir dans le dialogue avec les autres groupes intéressés. La boucle est ainsi bouclée et nous en revenons au souhait de dialogue mentionné au début du présent éditorial: quels sont les objectifs raisonnables que nous voulons atteindre en commun pour maintenir et promouvoir des soins médicaux de qualité? Quel niveau de qualité visons-nous? Et quelle est la valeur accordée à la qualité médicale par la société? Des questions qui ne pourront jamais assez être discutées ...

Dr Ludwig T. Heuss, p.-d., membre du Comité central, responsable du domaine DDQ

1 Peltenburg M, Kernen H, Schneider P, von Below GC, Waldis G, Vogel HA, et al. La qualité - une interaction de toutes les forces en présence dans le domaine médical. BMS 2005; 20:1216-20. 